\title{
Xerosis und Exsikkationsekzem unter Therapie eines metastasierten Adenokarzinoms mit monoklonalem Anti-VEGF-Antikörper Bevacizumab (Avastin ${ }^{\circledR}$ )
}

\author{
Xerosis and Asteatotic Eczema Following Treatment of Metastatic Adenocarcinoma with Monoclonal \\ Anti-VEGF Antibody Bevacizumab
}

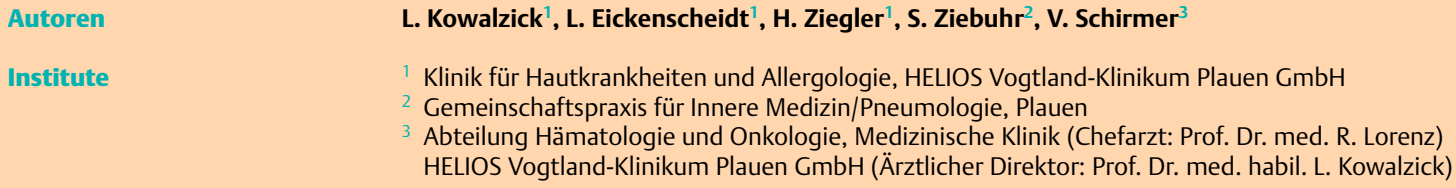

Institute

Klinik für Hautkrankheiten und Allergologie, HELIOS Vogtland-Klinikum Plauen GmbH

Gemeinschaftspraxis für Innere Medizin/Pneumologie, Plauen

Abteilung Hämatologie und Onkologie, Medizinische Klinik (Chefarzt: Prof. Dr. med. R. Lorenz)

HELIOS Vogtland-Klinikum Plauen GmbH (Ärztlicher Direktor: Prof. Dr. med. habil. L. Kowalzick)

\section{Bibliografie}

DOI $10.1055 / \mathrm{s}-2007-966838$

Akt Dermatol 2007; 33:

385-387 @ Georg Thieme

Verlag KG Stuttgart · New York ISSN 0340-2541

Korrespondenzadresse

Prof. Dr. med. habil.

Lutz Kowalzick

Klinik für Hautkrankheiten und Allergologie

HELIOS Vogtland-Klinikum

Plauen $\mathrm{GmbH}$

Postfach 100153

08505 Plauen

lutz.kowalzick@

helios-kliniken.de

\section{Zusammenfassung \\ $\nabla$}

Seit Kurzem wurden Inhibitoren des vaskulären Endothel-Wachstumsfaktors (VEGF) in der Therapie fortgeschrittener Karzinomerkrankungen, wie dem metastasierten kolorektalen Karzinom und dem metastasierten Mammakarzinom, zugelassen und werden in weiteren onkologischen Indikationen „off-label“ eingesetzt. Unter der Therapie mit Inhibitoren des epidermalen Wachstumsfaktors (EGF) tritt sehr häufig eine Symptomtrias kutaner Nebenwirkungen auf, die akneiforme Exantheme, Xerosis und Paronychien umfassen kann. Wir berichten über einen 64-jährigen Patienten mit metastasiertem Adenokarzi-

\section{Einleitung}

$\nabla$

Vor Kurzem wurde ein humanisierter monoklonaler Antikörper gegen VEGF, Bevacizumab $\left(\right.$ Avastin ${ }^{\circledR}$ ), zur First-line-Therapie des metastasierten kolorektalen Karzinoms und des metastasierten Mammakarzinoms zugelassen. Er wird jeweils in Kombination mit einer Chemotherapie mit 5-Fluorouracil (evtl. kombiniert mit Irinotecan) bzw. Paclitaxel eingesetzt. In klinischen Studien an mehreren hundert Patienten sprachen auf eine Kombinationstherapie mit Bevacizumab zwischen 10 und 20\% mehr Patienten mit kolorektalem Karzinom an als unter Chemotherapie allein [4]. Beim Mammakarzinom konnte das Gesamtüberleben gegenüber der Chemotherapie signifikant verlängert (verdoppelt) werden [8]. Darüber hinaus wird das Präparat im Rahmen individueller Heilversuche bei weiteren therapierefraktären onkologischen Indikationen eingesetzt. Bei Therapien mit EGF-Rezeptorantagonisten werden in über $80 \%$ kutane Nebenwirkungen beobachtet. Die häufigsten Nebenwirkungen an der Haut sind Akne mit 17-100\%, gefolgt von Hauttrockenheit (Xerosis, Desquamatio) mit ca. $26 \%$ nom mit okkultem Primärtumor und pulmonalen, pleuralen und ossären Metastasen sowie Verdacht auf Lebermetastasen, der mit Bevacizumab $\left(\right.$ Avastin $\left.^{\circledR}\right)$, einem monoklonalen Antikörper gegen den VEGF, behandelt wurde. Während der Therapie (2-wöchentliche i.v.-Applikation) entwickelte er binnen weniger Wochen eine generalisierte Xerosis mit vorwiegend palmoplantarer Manifestation eines Exsikkationsekzems. Diese Beobachtung könnte, neben anderen, darauf hinweisen, dass trotz fehlender Affinität zum EGFRezeptor, auch unter Therapie mit einem Antikörper gegen VEGF in Einzelfällen ähnliche kutane Nebenwirkungen auftreten wie unter Blockade des EGF-Signaltransduktionsweges.

sowie Nagelerkrankungen (Paronychie, Unguis incarnatus) mit ca. $12 \%[6,10]$.

Wir berichten hier über das Auftreten von Xerosis und Exsikkationsekzem auch bei einem 64-jährigen Patienten mit metastasiertem Adenokarzinom, der mit einer Kombinationstherapie mit einem VEGF-Antagonisten (Bevacizumab) und Docetaxel behandelt wurde.

\section{Kasuistik}

$\nabla$

Bei einem jetzt 64-jährigen Patienten wurden vor 4 Jahren pulmonale und pleurale Metastasen eines Adenokarzinoms diagnostiziert, wobei der Primärtumor nicht lokalisiert werden konnte (okkulter Primärtumor). Der Patient erhielt zweimal für 4 Monate eine Polychemotherapie mit Carboplatin und Vinorelbin. Zwischenzeitlich erfolgten Monochemotherapien für jeweils 4 Monate mit Gemcitabin bzw. Vinorelbin. Unter diesen Therapien kam es zeitweise zu Teilremissionen bzw. stabilem Erkrankungsverlauf. Zuletzt kam es aber zu einem Progress mit ossären und vermutlich auch hepatischen Metatasen, sodass 

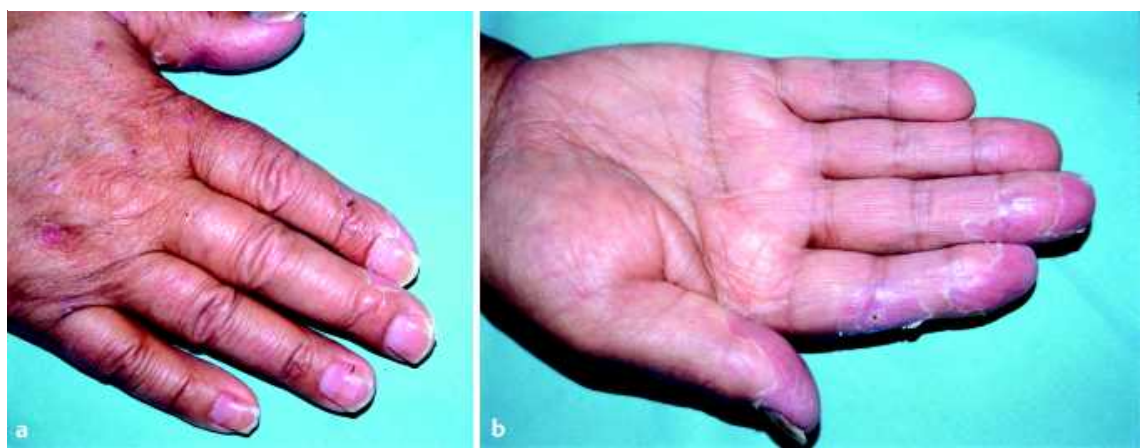

Abb. 1 a-d 64-jähriger Patient mit metastasiertem Adenokarzinom unter seit 3 Monaten eingesetzten Infusionen mit Bevacizumab, einem monklonalen Antikörper gegen den vaskulären Endothel Wachstumsfaktor (VEGF). Hauttrockenheit besonders palmoplantar $(\mathbf{b}, \mathbf{d})$ mit Pulpitis sicca (b) und schmerzhafter Rhagadenbildung im Fersenbereich (c) sowie beginnender Paronychie und Nagelwachstumsstörungen (a).

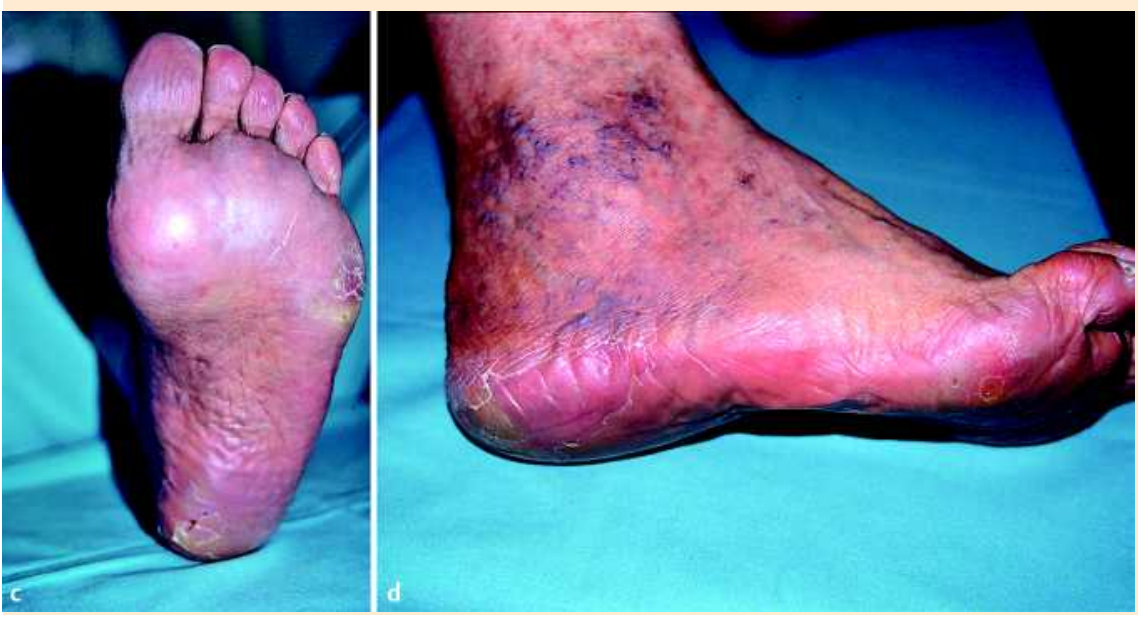

über 3 Monate eine Therapie mit Erlotinib, einem niedermolekularen Hemmer der EGF-Rezeptor-assoziierten Tyrosinkinase, durchgeführt wurde. Hierunter trat, wie zu erwarten, eine passagere generalisierte Xerosis auf, die sich nach Abbruch dieser Therapie zurückbildete. 4 Wochen nach Beginn einer Kombinationstherapie mit den VEGF-Antagonisten Bevacizumab sowie dem Taxan Docetaxel (Taxotere ${ }^{\circledR}$ ) beobachtete der Patient das erneute Auftreten von vermehrter Hauttrockenheit, diesmal betont an Händen und Füßen. Es fanden sich palmoplantar sowie insbesondere an den Hand- und Fußkanten flächige unscharf begrenzte Erytheme mit lamellöser und coloretteartig randständiger Schuppung ( $\bullet$ Abb. 1). Schmerzen gab der Patient dort nicht an, wohl aber geringen Juckreiz. Das mykologische Nativ- und Kulturpräparat fand sich an Händen und Füßen jeweils negativ. Wir verordneten rückfettende, harnstoffhaltige Externa.

\section{Diskussion \\ $\nabla$}

Seit wenigen Jahren werden monoklonale Antikörper gegen Wachstumsfaktoren und deren Rezeptoren sowie niedermolekulare Hemmer der für deren Signaltransduktion essenziellen Rezeptor-assoziierten Proteinkinasen in der onkologischen Therapie solider Tumoren eingesetzt. Bevacizumab ist ein Antikörper gegen VEGF und blockiert dessen Bindung an die spezifischen Rezeptoren VEGFR1 (Flt-1) und VEGFR2 (KDR), die vor allem auf Endothelzellen exprimiert werden. Tumorzellen können verstärkt VEGF bilden. Die Blockade von VEGF hemmt in der Folge die Tumorangionese und die Tumorprogression [1].

$\mathrm{Zu}$ den häufigeren (jeweils bei um die $20 \%$ der Patienten auftretenden ) Nebenwirkungen der Therapie mit Bevacizumab zählen Wundheilungsstörungen, Hypertonie, Proteinurie und Schleimhaut-Blutungen sowie Thromboembolien. Selten werden Ma-
gen-Darm-Perforationen oder ein reversibles posteriores Leukenzephalopathiesyndrom beobachtet [4].

Kürzlich wurde berichtet, dass akneiforme Exantheme, wie sie bei der Gabe von EGF-Rezeptor-Antagonisten regelmäßig auftreten, in Einzelfällen auch unter Therapie mit Bevacizumab auftreten können [3]. Eine weitere häufige Nebenwirkung von EGF-Rezeptor-Antagonisten sind Auftreten von Xerosis und Exsikkationsekzemen. Unser Fall würde dafür sprechen, dass diese im Einzelfall auch unter VEGF-Blockade auftreten können.

EGF bewirkt die autokrine bzw. parakrine Stimulation der VEGF Synthese durch Keratinozyten in vitro [2]. Es kann spekuliert werden, ob dieser Prozess auch vice versa stattfinden könnte und somit eine VEGF-Blockade zumindest lokal den EGF-Transduktionsweg behindern könnte. Denkbar wäre auch eine Bindung des Antikörpers gegen VEGF auch an EGF oder seinen Rezeptor. Schließlich könnten komplexere gegenseitige Beeinflussungen der verschiedenen Signalkaskaden unterschiedlicher Wachstumsfaktoren eine Rolle spielen.

Unter verschiedenen Zytostatika, unter anderem auch Taxanen, ist das häufigere Auftreten eines sogenannten palmoplantaren Erythrodysästhesie-Syndrom beobachtet worden [5]. Aber auch unter Sunitinib (Sutent ${ }^{\circledR}$ ) und Sorafenib (Nexavar $\left.{ }^{\circledR}\right)$, in Deutschland zugelassenen niedermolekularen Protein-Kinase-Inhibitoren [9], die unter anderem auch den VEGF-Rezeptor-Signaltransduktionsweg hemmen, wurden gleichfalls in bis zu 20\% der Fälle das palmoplantare Erythrodysästhesie-Syndrom beobachtet [7]. Aufgrund der deutlichen epidermalen Beteiligung, des eher schwachen Erythems und der fehlenden Dysästhesie würden wir unseren Fall diesem Syndrom jedoch nicht zurechnen wollen. Weitere Fallberichte sind wünschenswert, um die Häufigkeit des Auftretens typischer Nebenwirkungen, wie sie unter EGF-Blockade gesehen werden, auch unter Therapie mit VEGF-Antagonisten einschätzen zu können. 


\section{Abstract}

\section{Xerosis and Asteatotic Eczema Following Treatment of Metastatic Adenocarcinoma with Monoclonal Anti-VEGF Antibody Bevacizumab $\nabla$}

Recently, inhibitors of epidermal growth factor (VEGF) were approved by health authorities for treatment of metastatic carcinomas including colorectal and breast carcinoma. During therapy with inhibitors of epidermal growth factor, cutaneous side effects (e.g. acneiform eruptions, xerosis and paronychia) occur very frequently. We report on a 64 year old male patient with pulmonal, pleural, bone metastasis and suspected liver metastasis from an adenocarcinoma of unknown origin. During the therapy he developed within a few weeks generalized xerosis and palmoplantar pronounced asteatotic eczema. This observation, among others, could be a hint that, despite lacking affinity towards the EGF receptor, cutaneous side effects known from blockade of the EGF signal transduction could also occur during therapy with antibodies directed against VEGF in some cases.

\section{Literatur}

1 Ferrara $N$. Vascular endothelial growth factor: Basis science and clinical progress. Endocrine News 2004; 25: 581-611

2 Frank S, Hübner G, Breier G, Longaker MT, Greenhalgh DG, Werner S. Regulation of vascular endothelial growth factor expression in cultured keratinocytes. Implications for normal and impaired wound healing. J Biol Chem 1995; 270: 12 607-12613

3 Gotlib V, Khaled S, Lapko I, Mar N, Saif MH. Skin rash secondary to bevacizumab in a patient with advanced colorectal cancer and relation to response. Anti-Cancer Drugs 2006; 17: 1227-1229

4 Hurwitz H, Fehrenbacher L, Novotny W, Cartwright T, Hainsworth J, Heim W, Berlin J, Baron A, Griffing S, Holmgren E, Ferrara N, Fyve G, Rogers B, Ross R, Kabbinavar F. Bevacizumab plus irinotecan, fluorouracil, and leucovorin for metastatic colorectal cancer. N Engl J Med 2004; 350: $2332-2342$

5 Katoh M, Kadota M, Nishimura Y. A case of docetaxel-induced erythrodyseathesia. J Dermatol 2004; 31: 403-406

6 Kowazick L, Lohse C, Ziegler H, Busch FW. Schwere Akne unter Therapie eines metastasierten kolorektalen Karzinoms mit monoklonalem Anti-EGF-Rezeptor-Antikörper Cetuximab (Erbitux $\left.{ }^{\circledR}\right)$. Akt Dermatol 2004; 30: $474-477$

7 Kowalzick L. Nebenwirkungen an der Haut bei moderner Antikörpertherapie sowie bei Therapie mit Proteinkinase-Inhibitoren. Dt Dermatol 2007; 55: im Druck

8 Miller KD. E2100: a phase III trial of placitaxel versus placitaxel/bevacizumab for metastatic breast cancer. Clin Breast Cancer 2003; 3 : $421-422$

9 Müller-Tidow C, Krug U, Brunnberg U, Berdel WE, Serve H. Tyrosinkinasen als Ziel neuer onkologischer Therapien. Dtsch Arztebl 2007; 104: A $1312-1319$

10 Segaert $S$, van Cutsem E. Clinical signs, pathophysiology and management of skin toxicity during therapy with epidermal growth factor receptor inhibitors. Ann Oncol 2005; 16: 425-433 